\title{
(6) OPEN ACCESS \\ Advanced endoscopic ultrasound management techniques for preneoplastic pancreatic cystic lesions
}

\author{
Hafiz Muhammad Sharjeel Arshad, ${ }_{1}^{1}$ Sheila Bharmal, ${ }^{2}$ Deniz Guney Duman, ${ }^{3}$ \\ Suthat Liangpunsakul, ${ }^{4}$ Brian G Turner ${ }^{5}$
}

${ }^{1}$ Department of Internal

Medicine, University of Illinois at Chicago/Advocate Christ Medical Center, Oak Lawn, Illinois, USA

${ }^{2}$ Department of Gastroenterology, University of Minnesota, Minneapolis, Minnesota, USA

${ }^{3}$ Department of Gastroenterology, Marmara University, Istanbul, Turkey ${ }^{4}$ Division of

Gastroenterology and Hepatology, Department of Medicine, Indiana University School of Medicine, Indianapolis, Indiana, USA ${ }^{5}$ Ohio Gastroenterology Group, Columbus, Ohio, USA

\section{Correspondence to}

Dr Hafiz Muhammad

Sharjeel Arshad, Department of Internal Medicine, University of Illinois at Chicago/Advocate Christ Medical Center, $9840 \mathrm{~S}$ Pulaski Rd, Apt 204, Oak Lawn, IL 60453, USA; Sharjeel_169@hotmail.com

Accepted 1 August 2016 Published Online First 29 August 2016

Copyright (C) 2016 American Federation for Medical Research

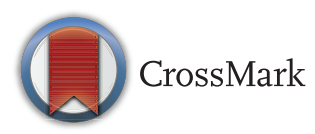

To cite: Arshad HMS, Bharmal S, Duman DG, et al. J Investig Med 2017;65:7-14.

\section{ABSTRACT}

Pancreatic cystic lesions can be benign, premalignant or malignant. The recent increase in detection and tremendous clinical variability of pancreatic cysts has presented a significant therapeutic challenge to physicians. Mucinous cystic neoplasms are of particular interest given their known malignant potential. This review article provides a brief but comprehensive review of premalignant pancreatic cystic lesions with advanced endoscopic ultrasound (EUS) management approaches. A comprehensive literature search was performed using PubMed, Cochrane, OVID and EMBASE databases. Preneoplastic pancreatic cystic lesions include mucinous cystadenoma and intraductal papillary mucinous neoplasm. The 2012 International Sendai Guidelines guide physicians in their management of pancreatic cystic lesions. Some of the advanced EUS management techniques include ethanol ablation, chemotherapeutic (paclitaxel) ablation, radiofrequency ablation and cryotherapy. In future, EUS-guided injections of drug-eluting beads and neodymium:yttrium aluminum agent laser ablation is predicted to be an integral part of EUS-guided management techniques. In summary, International Sendai Consensus Guidelines should be used to make a decision regarding management of pancreatic cystic lesions. Advanced EUS techniques are proving extremely beneficial in management, especially in those patients who are at high surgical risk.

\section{BACKGROUND}

Cystic lesions of the pancreas span a wide clinical spectrum and include inflammatory pseudocysts, non-mucinous (serous cystadenomas, solid pseudopapillary neoplasms) and mucinous cysts (mucinous cystadenomas (MCAs), mucinous cystadenocarcinomas and intraductal papillary mucinous neoplasms (IPMNs)). Ductal adenocarcinoma and neuroendocrine tumors are often included in cyst classification schemes, though these are largely solid pancreatic tumors that have undergone degenerative cystic change. ${ }^{1}$

Pancreatic cystic neoplasms represent benign, premalignant, and malignant lesions. The prevalence of pancreatic cysts in asymptomatic adults varies depending on the screening modality, ranging from $0.2 \%$ on the ultrasound to $\sim 20 \%$ on $\mathrm{MRI}^{2}{ }^{3}$ Higher rates are reported in the elderly and Asians. ${ }^{4}$ Generally, size $\geq 3 \mathrm{~cm}$, presence of a solid component, dilated pancreatic duct, growth of $>2 \mathrm{~mm} /$ year, presence of mural nodules, and suggestive cytology or biochemical analysis on fine needle aspiration (FNA) are associated with increased malignant potential..$^{5-8}$

Major management approaches include surgical resection, endoscopic techniques, and surveillance. Surgical resection or enucleation has long been considered the gold standard for management of premalignant and malignant lesions. The decision to resect a lesion is based on several factors: the presence or absence of symptoms, location of the lesion, probability of malignancy, and surgical risk of the patient. Further tools such as the Sendai International Consensus Guidelines have been developed to guide physicians in their decision-making. ${ }^{9}$ Surgical management is not without morbidity. Perioperative complication rates range between $20 \%$ and $40 \%$ and mortality approaches $2 \%$, even in robust surgical candidates. ${ }^{10-13}$ As such, endoscopic techniques have posed an attractive alternative to classic surgical interventions, particularly in patients with comorbidities or asymptomatic, indeterminate cystic lesions.

The increase in detection and tremendous clinical variability of pancreatic cysts has presented a significant therapeutic challenge to physicians. Mucinous cystic neoplasms are of particular interest due to their malignant potential. This review article focuses on the pathogenesis as well as the management of mucinous cystic neoplasms, due to their malignant potential.

\section{Mucinous cystic neoplasms}

Mucinous cystic neoplasms of the pancreas are cystic neoplasms which are composed of columnar, mucin-producing epithelium, supported by ovarian-type stroma. Clinically, most mucinous cystic neoplasms are discovered as incidental findings during imaging for another indication. Mucinous cystic neoplasms can compress adjacent organs and produce symptoms if they are large in size. These lesions are becoming an important point of discussion due to their potential for malignant transformation. 


\section{Review}

Mucinous cystic neoplasms can be further subdivided into three categories: MCAs, mucinous adenocarcinomas, and IPMNs.

\section{MCA and mucinous adenocarcinoma}

MCAs are premalignant and may progress to invasive mucinous cystadenocarcinoma. ${ }^{14}$ In one series, $10 \%$ of MCAs progressed to invasive adenocarcinoma at the time of resection. ${ }^{15} 16$ These macrocystic lesions are found mostly in women and have a propensity to form in the body and tail of the pancreas. ${ }^{17} 18$ MCAs are unilocular and typically do not communicate with the pancreatic duct. Lined by mucin-secreting cells, they often contain a highly cellular 'ovarian' stroma. Genetic mutations, including K-ras mutations, have been associated with the development of these lesions ${ }^{19}$ (figures 1 and 2).

\section{Intraductal papillary mucinous neoplasm}

IPMNs arise from the mucin-producing cells within the pancreatic duct. They are also considered to be premalignant and may progress to invasive adenocarcinoma. IPMNs are further classified into three types based on their site of origin: main duct (MD)-IPMN, branch duct (BD)-IPMN, and mixed type (arising from both ducts). ${ }^{17}$ MD-IPMNs

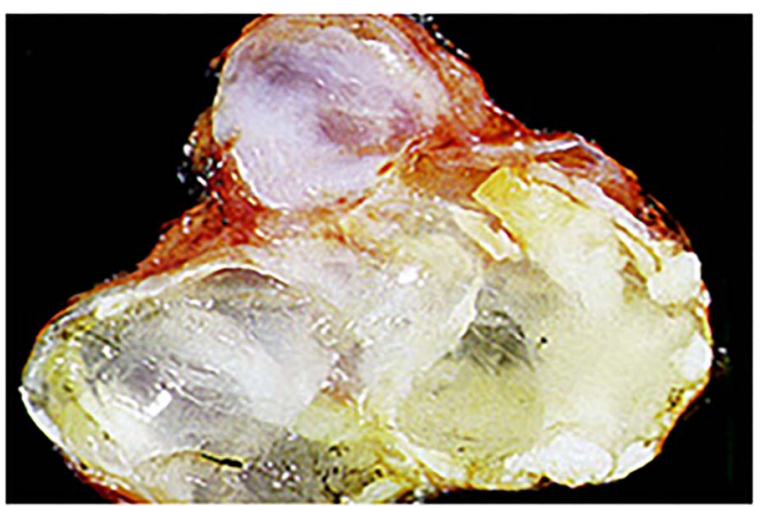

Figure 1 Gross appearance of a mucinous cystic lesion of the pancreas.

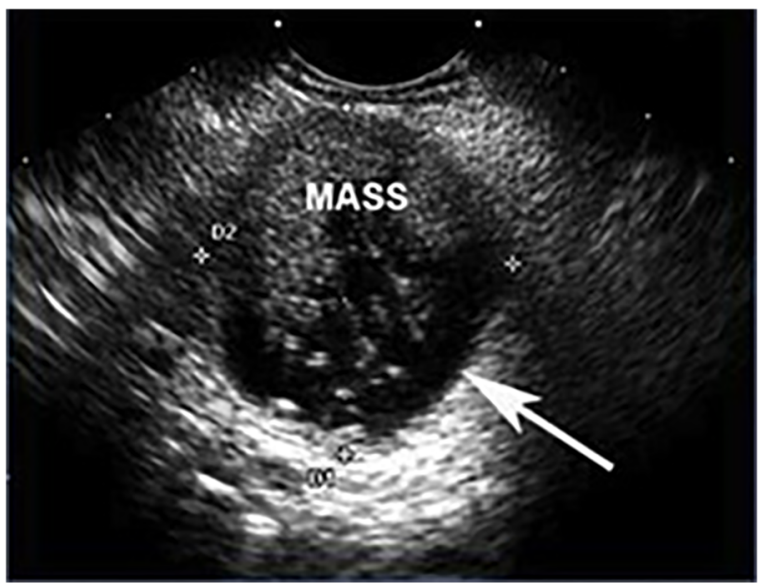

Figure 2 Endoscopic ultrasound of a malignant mucinous lesion with a mass filling the cystic space. The outer wall of the cyst is indicated by the white arrow. are more likely to be malignant with at least one-third harboring invasive cancer at the time of resection. IPMNs occur more commonly in elderly men and have a predilection for the head of the pancreas. Unlike MCAs, these lesions communicate with the pancreatic duct. The characteristic appearance on imaging or gross pathological evaluation is pancreatic ductal dilation secondary to papillary tumor growth. As in MCAs, the K-ras mutation has also been implicated in the development of IPMNs. ${ }^{1}$

\section{DIAGNOSTIC APPROACHES}

While most patients with pancreatic cystic lesions are asymptomatic, a small minority of patients may present with non-specific symptoms including recurrent abdominal discomfort, nausea, vomiting, and early satiety. Moreover, symptoms such as weight loss, jaundice, steatorrhea, and diabetes are more concerning and may be thought to herald malignancy. Physical examination is likely to be unrevealing, though in some cases, clinicians may note jaundice or a palpable abdominal mass. Given the limitations of a careful history and physical examination, radiographic and endoscopic imaging techniques have been employed to better characterize cystic lesions. The following is a detailed discussion of the imaging modalities available.

\section{CT scan}

CT scan is the most widely used initial imaging modality for diagnosis and provides detailed images of the pancreas and surrounding structures. Radiographic findings often reveal pathognomonic features sufficient for an accurate diagnosis and importantly aid in distinguishing malignant neoplasms. For example, detection of peripheral calcifications or a mural nodule by CT scan in a suspicious mucinous cystic neoplasm $(\mathrm{MCN})$ is highly suggestive of malignancy. In a study performed by Le Baleur Y et al, ${ }^{20}$ no malignant MCN had a diameter $<40 \mathrm{~mm}$. Thus, a diameter of $<40 \mathrm{~mm}$ is associated with low risk of malignancy (figures 3 and 4).

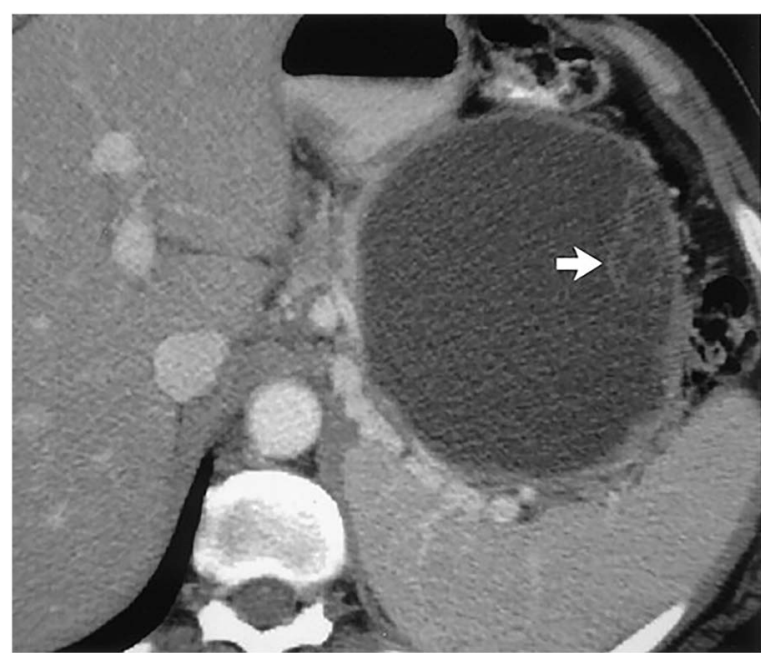

Figure 3 Contrast-enhanced CT scan showing mucinous cystadenoma. A large cystic tumor with small cysts clustered at its periphery (arrow). (Adapted with permission from: Kim YH et al). ${ }^{68}$ 


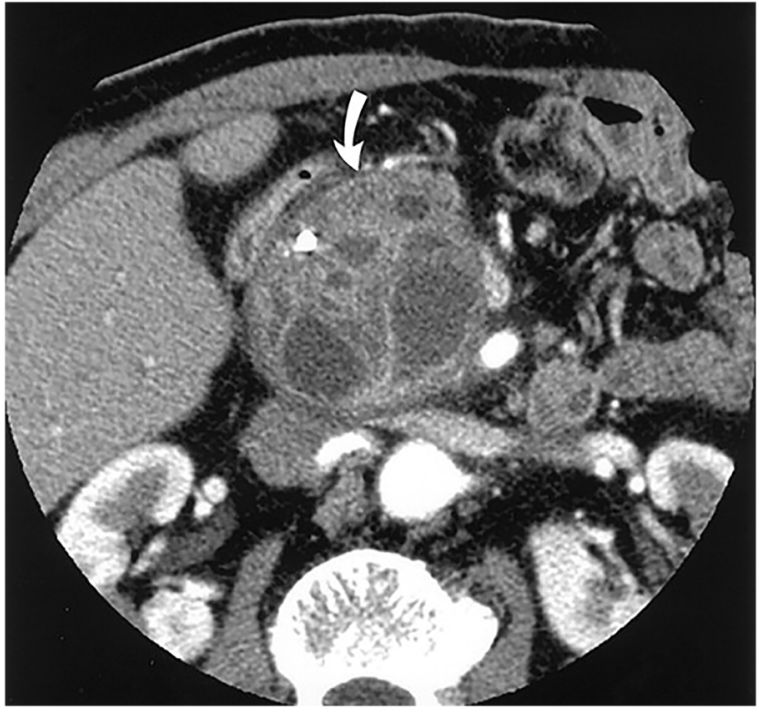

Figure 4 Contrast-enhanced CT scan showing branch duct IPMN. A cystic lesion can be seen with a markedly dilated side branch duct with wall thickening (arrow). (Adapted with permission from: $\mathrm{Kim}$ YH et al. $)^{68}$ IPMN, intraductal papillary mucinous neoplasm.

\section{MR cholangiopancreatography}

MR cholangiopancreatography (MRCP) has gained popularity because it allows for detailed visualization of the pancreatic ductal system and surrounding parenchyma without radiation exposure and invasive techniques. When applied to pancreatic cystic neoplasms, MCRP may aid in the identification of a ductal connection, small $\mathrm{BD}$ cysts or in the estimation of MD involvement. These factors influence diagnostic accuracy, cancer risk stratification and management strategies. Thus, MRCP use is very beneficial in all patients with IPMNs ${ }^{21}$ (figure 5).

\section{Endoscopic ultrasound with FNA}

When clinical and radiological indices are inadequate for reliable identification of pancreatic cystic structures, endoscopic ultrasound (EUS) in conjunction with FNA may be employed to provide valuable diagnostic information. Endoscopic imaging allows for detection of small lesions and may also yield staging information via examination of the surrounding vasculature. The addition of FNA improves diagnostic accuracy via analysis of molecular markers within the cystic fluid itself. Cystic fluid analysis should include tumor markers such as carcinoembryonic antigen (CEA) as well as amylase levels and careful cytological examination (figure 6).

Cystic fluid CEA levels are of particular interest. High CEA levels do not distinguish benign from malignant cysts and are not predictive of disease progression; ${ }^{22}$ however, studies have shown that higher CEA levels are associated with mucinous cysts. In fact, a CEA level of $192 \mathrm{ng} / \mathrm{mL}$ has a sensitivity approaching $80 \%$ for diagnosis. ${ }^{23}{ }^{24}$ Amylase levels are helpful in excluding pancreatic pseudocysts. A fluid amylase concentration of $<250 \mathrm{U} / \mathrm{L}$ indicates low risk of a pseudocyst. High pancreatic cyst fluid amylase values occur in pseudocysts and some mucin-producing cystic neoplasms; thus, they are non-specific. Cytological evaluation is the most important method in distinguishing benign from malignant cysts. ${ }^{22-25}$ In recent years, DNA

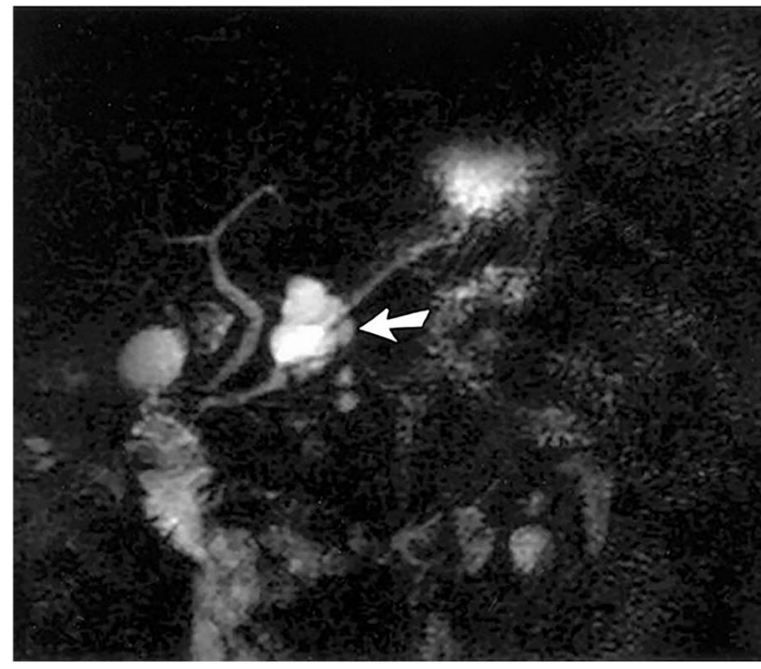

Figure 5 MR cholangiopancreatogram showing a lesion suspicious for IPMN. Image shows a cystic, grape-like pancreatic lesion. It is not clear whether there is a communication of the mass and the pancreatic duct. Endoscopic retrograde cholangiopancreatography (ERCP) would be useful in this situation. (Adapted with permission from: Kim YH, et al.) ${ }^{68}$ IPMN, intraductal papillary mucinous neoplasm.

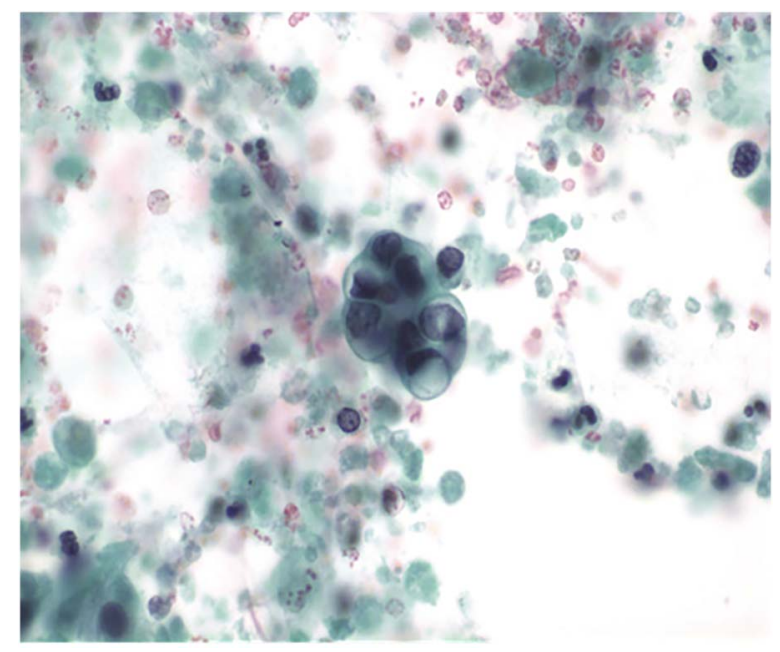

Figure 6 Cytology slide of IPMN aspirate from EUS-FNA. An intact cluster of moderately atypical epithelial cells with a single residual mucin vacuole. Even small numbers of such atypical cells warrant a suspicion of carcinoma. EUS, endoscopic ultrasound; FNA, fine needle aspiration; IPMN, intraductal papillary mucinous neoplasm.

analysis of cystic fluid is becoming a point of interest. A study performed by Nikiforova and colleagues showed that K-ras mutations are highly specific $(100 \%)$ but not very sensitive $(54 \%)$ for detection of mucinous differentiation of pancreatic cysts. K-ras mutation had a sensitivity of $67 \%$ for IPMNs and $14 \%$ for MCNs. ${ }^{26}$

\section{Needle-based confocal laser endomicroscopy}

Needle-based confocal laser endomicroscopy (nCLE) is a new diagnostic approach applied via EUS. It involves realtime imaging at the microscopic level using a sub-mm probe which can be introduced through an FNA needle. 
Napoleon and colleagues proposed new nCLE criteria for characterization of pancreatic cystic lesions (mucinous cystic neoplasm, cystic neuroendocrine neoplasms, pseuodocyst) based on histological correlation in a multicenter study. Histologically, mucinous cystic neoplasms consist of two distinct components: mucin-producing epithelium and fibrous tissue containing stroma composed of densely packed spindle cells with some large blood vessels. On nCLE, stroma was not clearly seen and the epithelial border was seen as a gray band. Histologically, a pseudocyst is seen as a fibroinflammatory tissue around necrotic adipocytes. On nCLE, a pseudocyst appears as a mixture of gray and black particles of various sizes $(10-40 \mu \mathrm{m})$. These particles represent inflammatory cells. A cystic neuroendocrine neoplasm is histologically characterized by a neoplastic monomorphic cell proliferation with variable organization and cellular structure. The cell arrangement can be trabecular, diffuse or nested. Fibrotic stroma surrounds these cellular arrangements. On nCLE, dark irregular clusters of compact cells representing neoplastic cells were seen which were surrounded by gray colored stroma.

Results showed an overall accuracy of $94 \%$ for detection of mucinous cystic lesions. Accuracy was found to be $87 \%$ for characterization of pseudocysts. ${ }^{27}$ Nakai and colleagues also assessed the diagnostic yield of combined nCLE and cystoscopy for diagnosis of pancreatic cystic neoplasms. Mucin on cystoscopy and papillary projections or black rings on nCLE were found as characteristic features of MCNs. This study described that when used alone, EUS had a sensitivity of $53 \%$ and specificity of $67 \%$ for diagnosis of MCNs. When nCLE was used for diagnosis, sensitivity increased to $80 \%$ and specificity to $100 \%$. Use of cytology further improved sensitivity to $90 \%$. Combined use of nCLE and cystoscopy for diagnosis of MCNs produced results with $100 \%$ sensitivity and specificity. ${ }^{28}$

\section{MANAGEMENT GUIDELINES}

The 2012 Sendai International Consensus Guidelines were released to guide physicians in their management of MCNs and IPMNs. They recommend CT or MRCP for all cysts $\geq 1 \mathrm{~cm}$. On the basis of these imaging techniques, patients may be classified into one of two groups, high-risk stigmata or worrisome features. High-risk stigmata include evidence of an enhanced solid component of cysts, $\mathrm{MD} \geq 10 \mathrm{~mm}$ or the presence of obstructive jaundice. Cysts with high-risk stigmata should be resected in patients medically fit for surgery. In these patients, EUS is considered optional, but may provide useful information preoperatively. Worrisome features include but are not limited to cysts $\geq 3 \mathrm{~cm}$, thickened or enhanced cyst walls, MD size between 5 and $9 \mathrm{~mm}$, and abrupt change in appearance and lymphadenopathy. EUS is subsequently recommended for all cysts with worrisome features or for cysts $>3 \mathrm{~cm}$ without these features. EUS confirmation of a mural nodule, MD involvement or suspicious cytology is an indication for surgical resection. Additionally, EUS can be considered in smaller cysts without worrisome features but is not required. ${ }^{9}$

\section{ADVANCED EUS MANAGEMENT APPROACHES Ethanol ablation}

Ablative ethanol therapy of cystic lesions has long been regarded as a safe, effective and minimally invasive management strategy, particularly when applied to hepatic, renal, splenic, endometrial and thyroid cysts. ${ }^{29-36}$ For pancreatic cysts, this therapeutic option poses an attractive alternative to classic surgical approaches, especially in those patients with numerous preoperative comorbidities. Ethanol is an inexpensive, low-viscosity, and widely available agent thought to induce cell membrane lysis, protein denaturation, and vascular occlusion within minutes of injection. $^{37-39}$ Furthermore, when applied specifically to pancreatic cystic lesions, the ethanol injection is thought to reduce the risk of neoplasm by eliminating the source of neoplasia, the cyst epithelium. ${ }^{40}$

Ethanol ablation is best performed by an experienced endoscopist given the complex morphology of most pancreatic cysts. Pancreatic cysts are visualized using either a radial scanning echoendoscope or a curvilinear array echoendoscope. Cyst aspiration often occurs via a transgastric or transduodenal approach and may be challenging, particularly in cases of mucinous cysts given the viscous nature of the aspirate. Evacuation of cyst fluid provides useful diagnostic information, creates space within the cavity for the ablative agent and increases the surface area of exposure to the ablative agent. ${ }^{41}$ After subtotal evacuation, ethanol is injected and the cyst cavity is lavaged for 3-5 min, alternately filling and emptying. The concentration of ethanol varies, sometimes approaching 99\% depending on the endoscopist's preference, and does not appear to correlate with successful cyst resolution. At the end of the lavage, the injected ethanol is mostly evacuated, leaving only enough solution to outline the cyst cavity. Care is taken throughout the procedure to ensure that the needle tip is maintained within the cyst, to avoid parenchymal injury or a leak in the wall. Once the procedure is complete, the needle is removed from the cyst cavity. ${ }^{42}$ This algorithm becomes more complex when applied to morphologically complex cysts, defined as larger, thick walled, multiloculated cysts. ${ }^{39}{ }^{42}$ In these instances, a single needle pass may not be sufficient to provide adequate delivery of the ablative agent and the angle of introduction should be optimized such that the maximum number of locules are traversed. ${ }^{39} 42$ Even one missed locule may result in cyst regeneration and treatment failure.

Procedural success has been well documented in the literature with overall resolution on imaging ranging from $33 \%$ to $38 \%$ at a follow-up period of 3-13 months. ${ }^{40}$ In the largest multicenter, randomized, controlled, doubleblind study examining this subject matter, DeWitt and colleagues compared EUS-guided saline lavage to ethanol lavage. They concluded that ethanol lavage resulted in a greater decrease in cyst surface area and noted overall CT-defined resolution in 33\% of patients at 3-4 months after ablation. ${ }^{42}$ Concerns related to long-term procedural success prompted DeWitt and colleagues to perform a subsequent longitudinal evaluation of patients from the original study cohort. All nine patients with initial CT-defined resolution demonstrated no evidence of cyst recurrence on repeat cross-sectional imaging at a median of 26 months postablation. ${ }^{42}$ The results of this study suggest a durable ablative response, effectively reducing the likelihood of cyst reaccumulation and subsequent malignant transformation. Park and colleagues demonstrated that the success rate of ethanol ablation was different depending on cystic fluid 
analysis (serous cystic neoplasm, 58\%; mucinous cystic neoplasm, 50\%; IPMN, 11\%; uncategorized cysts, 39\%; $\mathrm{p}<0.0001$ ). On the basis of these results, ethanol ablation produced disappointing results for treatment of IPMNs. ${ }^{43}$

Complications are uncommon and occur in some cases, most commonly abdominal pain (18\%) and pancreatitis $(3 \%) .{ }^{43}$ Pancreatitis may occur in the presence of a communication between the cyst and main pancreatic duct. In this instance, flow of the ablative agent may traverse the main pancreatic duct, resulting in local inflammation as well as a diminished ablative effect. Additionally, intracystic bleeding has been reported in rare cases. Interestingly, a risk of clinical ethanol intoxication also exists and has been documented in patients undergoing ethanol ablation of thyroid cysts. $^{44}$

\section{Ablation with chemotherapeutic agents}

A second ablative agent, paclitaxel, has been applied in the management of pancreatic cystic neoplasms as well as in tumors of the lung and brain. ${ }^{33}{ }^{34}$ Paclitaxel is a commonly used chemotherapeutic drug that works by inhibiting cell processes that are dependent on microtubules, resulting in cessation of cell division and induction of apoptosis. This agent is hydrophobic and highly viscous, though its consistency varies somewhat depending on the composition of its co-solvent. ${ }^{45}$ A localized, rather than systemic, application of paclitaxel has gained significant favor, theoretically minimizing the toxicities of therapy while also increasing therapeutic concentrations within the cyst itself. ${ }^{46}$

In practice, paclitaxel and ethanol are thought to have a synergistic ablative effect; thus, the combined use of these two agents has been well described in the literature. Ethanol lavage causes distortion of the cyst epithelial lining and paclitaxel exerts secondary tumorous inhibition on the injured tissue. $^{344247}$ The technique applied is similar to the methods described above for ethanol ablation. Ethanol is first injected into a collapsed cyst, followed by a 3-5 min lavage of the aspirate. The ethanol solution is subsequently reaspirated and paclitaxel is injected into the empty cyst cavity. The viscosity of the solution is thought to be of mixed utility, reducing the risk of leakage through the puncture site but also complicating the injection technique itself. $^{45}$ The needle is retrieved and the chemotherapeutic agent remains within the cyst to exert its effect. A study showed that complete resolution was achieved in $62 \%$ of patients with a 2 -year follow-up. ${ }^{41}$ In 2014 , DeWitt and colleagues published a study showing a combined management approach with ethanol and paclitaxel. Their results demonstrated complete response in 50\%, partial response in $25 \%$ and a persistent cyst in $25 \%$ of patients. Adverse events included abdominal pain in $13 \%$, pancreatitis in $10 \%$, peritonitis in $3 \%$ and gastric wall cyst in $3 \%$. $^{48}$

\section{Radiofrequency ablation}

Radiofrequency ablation (RFA) is a well-established procedure that has been applied to cystic lesions of the kidney, thyroid, and pancreas, among others. ${ }^{49} 50$ Under endoscopic guidance, this technique allows targeted delivery of electrical therapy, producing initial coagulative necrosis and eventual fibrotic changes in surrounding tissues. ${ }^{49}$

RFA uses a high-frequency $(460-500 \mathrm{kHz})$ alternating current that is delivered to a targeted lesion through a thin needle which is inserted through the working channel of an endoscope. This needle is electrically insulated except for its terminal $1-3 \mathrm{~cm}$. A current produces resistive friction in the tissue that is converted into heat, similar to the way heat is generated from an electrical resistor in a circuit. The current is completed with grounding pads placed on the patient's back and thighs. RFA can predictably produce an ablation area of $3 \mathrm{~cm}$ at a single application, so cystic lesions $>3 \mathrm{~cm}$ may require more than one deployment of the needle electrode. ${ }^{51}$ This technology has only recently been adopted by endoscopists; thus, efficacy data are limited. Initial studies demonstrated that this technology is feasible, effective and safe when applied to the porcine pancreas. ${ }^{51}$ Pai and colleagues used RFA ablation for management of pancreatic cystic lesions in six patients. Postprocedural imaging 3-6 months after treatment found complete resolution of the cystic lesion in two patients and $48.4 \%$ reduction in the size of the cystic lesion in three patients. No major complications were observed within 48 hours of the procedure. Two patients reported abdominal pain. ${ }^{52}$

The application of RFA in unresectable pancreatic cancer both in human and animal models demonstrates significant morbidity associated with its use. ${ }^{53}$ Complications such as pancreatitis, perforation, or thermal injury to the bile duct, pancreatic parenchyma, and vessels occurred. ${ }^{53}{ }^{54}$ Cooling of adjacent tissue and real-time visualization of the target and surrounding structures has reduced procedure-related complications $^{54-57}$ (figure 7).

\section{Cryotherapy}

Cryotherapy may also be applied to pancreatic cystic neoplasms and has demonstrated utility in neoplasms of the liver, prostate, lung and breast. ${ }^{58}$ This technology is gaining increasing popularity, particularly when used with adjunctive therapies such as irradiation, chemotherapeutic drugs, apoptotic promoters and immunological agents. ${ }^{58} 59$ In contrast to the thermal injury generated via RFA, cryotherapy induces damage via extreme cold produced by the application of liquid nitrogen or argon gas. In vitro studies on cancer cells and in vivo animal studies on implanted cells suggest that temperature of at least $-4^{\circ} \mathrm{C}$ is necessary

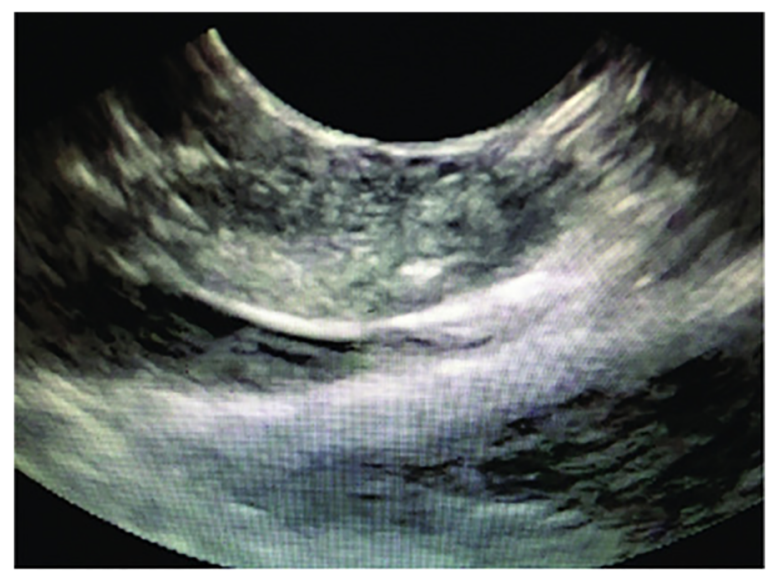

Figure 7 EUS view of the EUS-RFA probe. EUS, endoscopic ultrasound; RFA, radiofrequency ablation. 


\section{Review}

for destruction of abnormal cells. ${ }^{58-60}$ Initial cryoinjury is related to the combination of ice-associated damage and cellular osmotic shock during the freeze-thaw process while delayed injury arises from the progressive failure of microcirculation that leads to vascular stasis and necrosis. ${ }^{61}$

Cryotherapy may be performed under real-time guidance using EUS, CT scan or MRI. This discussion will focus on
EUS-guided cryoablation. A 2 or $3 \mathrm{~mm}$ hollow instrument called a cryoprobe is brought in contact with abnormal tissue via the working channel of the endoscope. For larger lesions, multiple probes may be used. Refrigerants are usually forced through the device under pressure to allow for quick delivery and to preserve the flexibility of the cryoprobe. Two cycles of freezing/thawing are performed with subsequent targeted tissue injury occurring. ${ }^{62}$

Table 1 Comparison of preneoplastic pancreatic cysts (MCN and IPMN) with pseudocyst

\begin{tabular}{|c|c|c|c|c|}
\hline & & Pseudocyst & $\mathrm{MCN}$ & IPMN \\
\hline Location & & Anywhere & Primarily body and tail & Head, body and tail \\
\hline EUS features & & $\begin{array}{l}\text { Primarily unilocular, anechoic or contains } \\
\text { debris }\end{array}$ & Unilocular or oligolocular & $\begin{array}{l}\text { Multiple cysts communicating with } \\
\text { pancreatic duct, dilated pancreatic } \\
\text { duct }\end{array}$ \\
\hline $\begin{array}{l}\text { Cystic fluid } \\
\text { analysis }\end{array}$ & $\begin{array}{l}\text { CEA } \\
\text { Amylase } \\
\text { Cytology }\end{array}$ & $\begin{array}{l}\text { Low } \\
\text { High } \\
\text { Inflammatory cells }\end{array}$ & $\begin{array}{l}\text { High } \\
\text { Usually low but may be high } \\
\text { Extracellular mucin, mucinous } \\
\text { epithelial } \\
\text { cells and atypical cells may be seen }\end{array}$ & $\begin{array}{l}\text { High } \\
\text { High } \\
\text { Extracellular mucin, mucinous } \\
\text { epithelial } \\
\text { cells and atypical cells may be seen }\end{array}$ \\
\hline
\end{tabular}

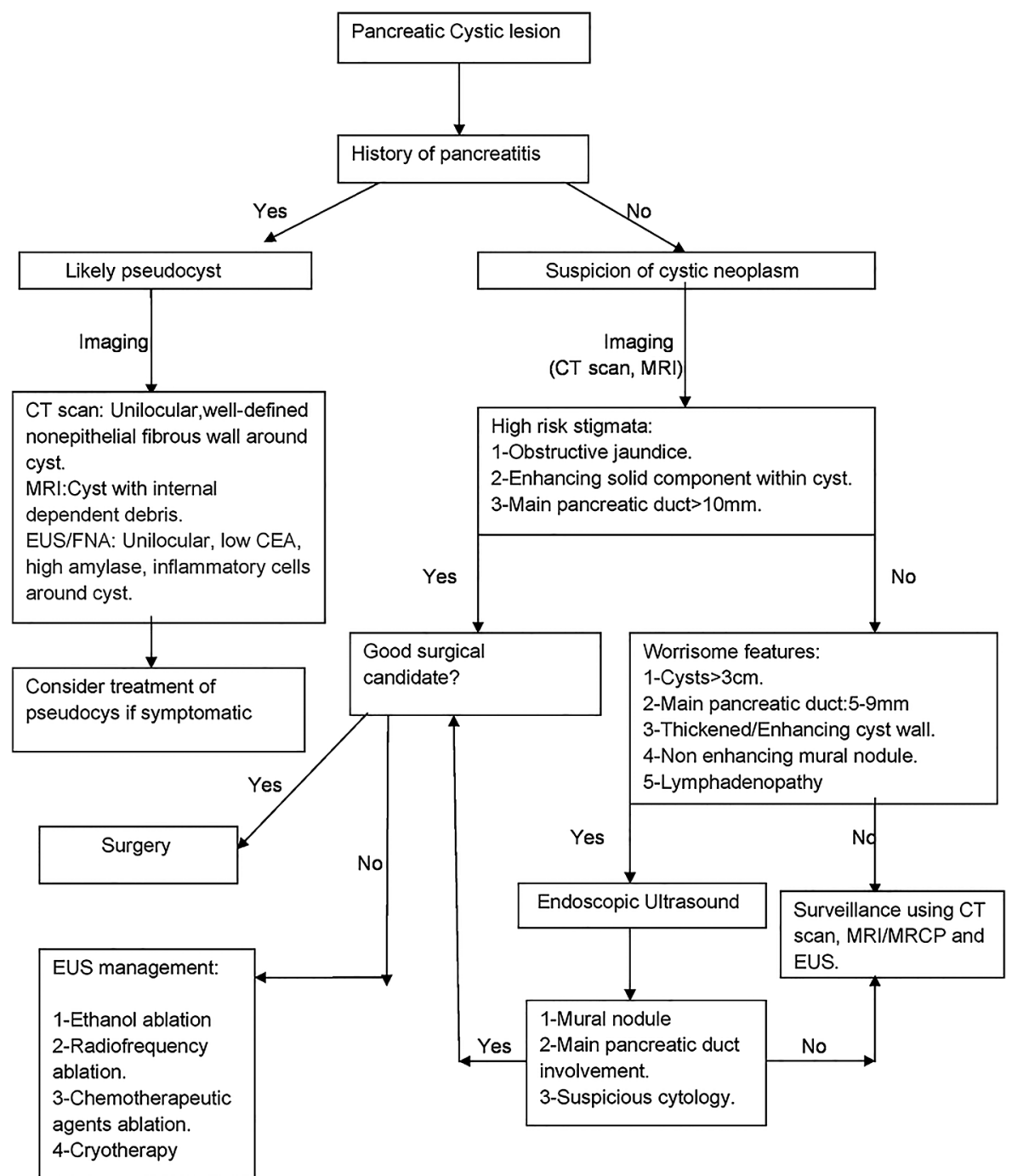

Figure 8 Schematic with a brief summary of management of cystic pancreatic lesions. CEA, carcinoembryonic antigen; EUS, endoscopic ultrasound; FNA, fine needle aspiration; MRCP, MR cholangiopancreatography. 
When applied to the pancreas, this technique is well known when used in conjunction with brachytherapy or iodine-125 seed implantation. Iodine-125 is an isotope which may be implanted at the border of the neoplasm where temperatures are suboptimal for complete tissue injury. Seed implantation provides local delivery of $\gamma$ radiation and induction of apoptosis. Xu et al ${ }^{61}$ examined 49 patients who underwent iodine- 125 seed implantation after percutaneous cryosurgery and found improvements in median survival when compared with cryotherapy alone. Other well-known combination therapies include RFA and cryogenic cooling using a bipolar cryotherm probe endoscopically. Arcidiacono $e^{a}$ al $^{62}$ conducted a cohort study of 16 patients and demonstrated feasibility and safety of this technology in the pancreas. Endoscopic cryotherapy of pancreatic cysts is a relatively new technology; thus, efficacy data are limited.

Complications of cryoablation include those caused by hypothermic damage to adjacent normal tissue, damage along the probe track and seeding of neoplastic cells during probe removal. Incomplete destruction of cells and persistence of neoplastic tissue is possible, particularly within the border zones where temperatures may be somewhat warmer than target. In rare cases, perforation and strictures may occur. ${ }^{62}$

\section{FUTURE DIRECTIONS}

\section{EUS-guided injection of drug-eluting beads}

EUS-guided injection of drug-eluting beads loaded with chemotherapeutic agents such as doxorubicin, mitoxantrone, and irinotecan was first described in its application to colorectal liver metastasis. Recently, this technique has been applied to the pancreas, targeting hepatic metastasis and peritoneal implants of pancreatic origin. ${ }^{63-65}$ These beads are biocompatible polyvinyl-alcohol hydrogel beads which allow a slow and sustained release of the drug within a targeted area. Current research demonstrates the utility of this EUS-guided therapy when applied directly to neoplasms of the pancreas in animal models. Karaca et $a l^{66}$ demonstrated the feasibility and safety of EUS-guided irinotecan-loaded beads in a porcine model. Additional investigation is needed to further guide use in patients with pancreatic cystic neoplasms.

\section{EUS-guided neodymium:yttrium aluminum agent laser ablation}

Laser ablation with a neodymium:yttrium aluminum agent (Nd:YAG) laser is being used in the management of hepatocellular carcinoma, liver metastasis in colorectal cancer and malignant thyroid nodules. These lasers typically emit light with a wavelength in the infrared spectrum with energy output sufficient for induction of tissue necrosis. DiMatteo and colleagues assessed the feasibility of the Nd:YAG laser for pancreatic tissue necrosis in an animal model. The $\mathrm{Nd}$ : YAG laser $(1.064 \mathrm{~nm})$ with an output power of 2 and $3 \mathrm{~W}$ and a total delivery energy of 500 and $1000 \mathrm{~J}$ in continuous mode was used in this study. Tissue necrosis of pancreatic parenchyma was observed in all animal models on histological examination. No significant technical difficulties or side effects were encountered. ${ }^{67}$ This technology remains investigational and future studies of the Nd:YAG laser are needed prior to implementation.

\section{CONCLUSIONS}

Preneoplastic pancreatic cystic lesions are clinically and pathologically diverse with a variable disease course. Major management approaches include surgical resection, endoscopic techniques, and surveillance. Endoscopic therapeutic options are vast, minimally invasive, and enable targeted, individualized therapy for patients who should not undergo surgical resection. Additional investigation is needed to evaluate the efficacy and adverse effects of endoscopic treatment approaches in place.

Table 1 shows a brief summary of differences between preneoplastic cystic lesions and pseudocyst.

Below is a simplified algorithm for management of cystic lesions of the pancreas with special focus toward preneoplastic cystic lesions management (figure 8).

\section{Competing interests None declared.}

Provenance and peer review Not commissioned; externally peer reviewed.

Open Access This is an Open Access article distributed in accordance with the Creative Commons Attribution Non Commercial (CC BY-NC 4.0) license, which permits others to distribute, remix, adapt, build upon this work noncommercially, and license their derivative works on different terms, provided the original work is properly cited and the use is non-commercial. See: http:/l creativecommons.org/licenses/by-nc/4.0/

\section{REFERENCES}

1 Enestvedt BK, Ahmad N. To cease or 'de-cyst'? The evaluation and management of pancreatic cystic lesions. Curr Gastroenterol Rep 2013;15:348.

2 Ikeda M, Sato T, Morozumi A, et al. Morphologic changes in the pancreas detected by screening ultrasonography in a mass survey, with special reference to main duct dilatation, cyst formation, and calcification. Pancreas 1994;9:508-12.

3 Zhang XM, Mitchell DG, Dohke $M$, et al. Pancreatic cysts: depiction on single-shot fast spin-echo MR images. Radiology 2002;223:547-53.

4 Laffan TA, Horton KM, Klein AP, et al. Prevalence of unsuspected pancreatic cysts on MDCT. AJR Am J Roentgenol 2008;191:802-7.

5 Rodriguez JR, Salvia R, Crippa S, et al. Branch-duct intraductal papillary mucinous neoplasms: observations in 145 patients who underwent resection. Gastroenterology 2007;133:72-9; quiz 309-310.

6 Scheiman JM, Hwang JH, Moayyedi P. American Gastroenterological Association technical review on the diagnosis and management of pancreatic cysts. Gastroenterology 2015;148:824-48.

7 Kang MJ, Jang JY, Kim SJ, et al. Cyst growth rate predicts malignancy in patients with branch duct intraductal papillary mucinous neoplasms. Clin Gastroenterol Hepatol 2011;9:87-93.

8 Kubo H, Chijiiwa Y, Akahoshi K, et al. Intraductal papillary mucinous tumors of the pancreas differential diagnosis between benign and malignant tumors by endoscopic ultrasonography. Am J Gastroenterol 2001;96:1429-34.

9 Tanaka M, Chari S, Adsay V, et al. International consensus guidelines for management of intraductal papillary mucinous neoplasms and mucinous cystic neoplasms of the pancreas. Pancreatology 2006;6:17-32.

10 Allen PJ, D'Angelica M, Gonen $M$, et al. A selective approach to the resection of cystic lesions of the pancreas: results from 539 consecutive patients. Ann Surg 2006;244:572-82.

11 Kiely JM, Nakeeb A, Komorowski RA, et al. Cystic pancreatic neoplasms: enucleate or resect? J Gastrointest Surg 2003;7:890-7.

12 Horvath KD, Chabot JA. An aggressive resectional approach to cystic neoplasms of the pancreas. Am J Surg 1999;178:269-74.

13 Goh BK, Tan YM, Cheow PC, et al. Cystic lesions of the pancreas: an appraisal of an aggressive resectional policy adopted at a single institution during 15 years. Am J Surg 2006;192:148-54.

14 Jones NB, Hatzaras I, George N, et al. Clinical factors predictive of malignant and premalignant cystic neoplasms of the pancreas: a single institution experience. HPB (Oxford) 2009;11:664-70.

15 Valsangkar NP, Morales-Oyarvide V, Thayer SP, et al. 851 resected cystic tumors of the pancreas: a 33-year experience at the Massachusetts General Hospital. Surgery 2012;152(3 Suppl 1):S4-12.

16 Crippa S, Fernandez-Del Castillo C, Salvia R, et al. Mucin-producing neoplasms of the pancreas: an analysis of distinguishing clinical and epidemiologic characteristics. Clin Gastroenterol Hepatol 2010;8:213-19. 
17 Crippa S, Salvia R, Warshaw AL, et al. Mucinous cystic neoplasm of the pancreas is not an aggressive entity: lessons from 163 resected patients. Ann Surg 2008;247:571-9.

18 Turner BG, Brugge WR. Cysts and pseudocysts of the pancreas. In: Textbook of clinical gastroenterology and hepatology.

19 Wilentz RE, Albores-Saavedra J, Zahurak M, et al. Pathologic examination accurately predicts prognosis in mucinous cystic neoplasms of the pancreas. Am J Surg Pathol 1999;23:1320-7.

20 Le Baleur Y, Couvelard A, Vullierme MP, et al. Mucinous cystic neoplasms of the pancreas: definition of preoperative imaging criteria for high-risk lesions. Pancreatology 2011:11:495-9.

21 Waters JA, Schmidt CM, Pinchot JW, et al. CT vs MRCP: optimal classification of IPMN type and extent. J Gastrointest Surg 2008;12:101-9.

22 Cizginer S, Turner BG, Bilge AR, et al. Cyst fluid carcinoembryonic antigen is an accurate diagnostic marker of pancreatic mucinous cysts. Pancreas 2011;40:1024-8.

23 Nagula S, Kennedy T, Schattner MA, et al. Evaluation of cyst fluid CEA analysis in the diagnosis of mucinous cysts of the pancreas. J Gastrointest Surg 2010;14:1997-2003.

24 Brugge WR, Lewandrowski K, Lee-Lewandrowski $\mathrm{E}$, et al. Diagnosis of pancreatic cystic neoplasms: a report of the cooperative pancreatic cyst study. Gastroenterology 2004;126:1330-6.

25 Genevay M, Mino-Kenudson M, Yaeger K, et al. Cytology adds value to imaging studies for risk assessment of malignancy in pancreatic mucinous cysts. Ann Surg 2011;254:977-83.

26 Nikiforova MN, Khalid A, Fasanella KE. Integration of KRAS testing in the diagnosis of pancreatic cystic lesions: a clinical experience of 618 pancreatic cysts. Mod Pathol 2013;26:1478-87.

27 Napoleon B, Lemaistre Al, Pujol B, et al. In vivo characterization of pancreatic cystic lesions by needle-based confocal laser endomicroscopy ( $\mathrm{nCLE}$ ): proposition of a comprehensive nCLE classification confirmed by an external retrospective evaluation. Surg Endosc 2015;30:2603-12.

28 Nakai $Y$, Iwashita T, Park do H, et al. Diagnosis of pancreatic cysts: EUS-guided, through-the-needle confocal laser-induced endomicroscopy and cystoscopy trial: DETECT study. Gastrointest Endosc 2015;81:1204-14.

29 Hanna RM, Dahniya MH. Aspiration and sclerotherapy of symptomatic simple renal cysts: value of two injections of a sclerosing agent. AJR Am J Roentgenol 1996;167:781-3.

30 Larssen TB, Viste A, Jensen DK, et al. Single-session alcohol sclerotherapy in benign symptomatic hepatic cysts. Acta Radiol 1997;38:993-7.

31 Gelczer RK, Charboneau JW, Hussain S, et al. Complications of percutaneous ethanol ablation. J Ultrasound Med 1998;17:531-3.

32 Gan SI, Thompson CC, Lauwers GY, et al. Ethanol lavage of pancreatic cystic lesions: initial pilot study. Gastrointest Endosc 2005;61:746-52.

33 Celikoglu SI, Celikoglu F, Goldberg EP. Endobronchial intratumoral chemotherapy (EITC) followed by surgery in early non-small cell lung cancer with polypoid growth causing erroneous impression of advanced disease. Lung Cancer 2006;54:339-46.

34 Hader WJ, Steinbok P, Hukin J, et al. Intratumoral therapy with bleomycin for cystic craniopharyngiomas in children. Pediatr Neurosurg 2000;33:211-18.

35 Chang KJ, Nguyen PT, Thompson JA, et al. Phase I clinical trial of allogeneic mixed lymphocyte culture (cytoimplant) delivered by endoscopic ultrasound-guided fine-needle injection in patients with advanced pancreatic carcinoma. Cancer 2000;88:1325-35.

36 Hecht JR, Bedford R, Abbruzzese JL, et al. A phase I/II trial of intratumoral endoscopic ultrasound injection of ONYX-015 with intravenous gemcitabine in unresectable pancreatic carcinoma. Clin Cancer Res 2003;9:555-6.

37 DeWitt J, McGreevy K, Schmidt CM, et al. EUS-guided ethanol versus saline solution lavage for pancreatic cysts: a randomized, double-blind study. Gastrointest Endosc 2009;70:710-23.

38 Van der Waaij LA, van Dullemen HM, Porte RJ. Cyst fluid analysis in the differential diagnosis of pancreatic cystic lesions: a pooled analysis. Gastrointest Endosc 2005;62:383-9.

39 Brugge WR. Management and outcomes of pancreatic cystic lesions. Dig Liver Dis 2008;40:854-9.

40 DiMaio CJ, DeWitt JM, Brugge WR. Ablation of Pancreatic Cystic lesions: the use of multiple endoscopic ultrasound guided ethanol lavage sessions. Pancreas 2011;40:664-8.

41 Oh HC, Seo DW, Song TJ, et al. Endoscopic ultrasonography guided ethanol lavage with paclitaxel injection treats patients with pancreatic cysts. Gastroenterology 2011;140:172-9.
42 DeWitt J, DiMaio CJ, Brugge WR. Long term follow-up of pancreatic cysts that resolve radiologically after EUS-guided ethanol ablation. Gastrointest Endosc 2010:72:862-6.

43 Park JK, Song BJ, Ryu JK, et al. Clinical outcomes of endoscopic ultrasonography-guided pancreatic cyst ablation. Pancreas 2016;45:889-94.

44 Oh HC, Brugge WR. EUS guided pancreatic cyst ablation: a critical review (with video). Gastrointest Endosc 2013;77:526-33.

45 Rowinsky EK, Donehower RC. Paclitaxel (taxol). N Engl J Med 1995:332:1004-14.

46 Verna EC, Dhar V. Endoscopic ultrasound-guided fine needle injection for cancer therapy. The evolving role of therapeutic endoscopic ultrasound. Therap Adv Gastroenterol 2008;1:103-9.

47 Oh HC, Seo DW, Kim SC, et al. Septated cystic tumors of the pancreas. Is it possible to treat them by endoscopic ultrasonography-guided intervention? Scand J Gastroenterol 2009;44:242-7.

48 DeWitt JM, Al-Haddad M, Sherman S, et al. Alterations in cyst fluid genetics following endoscopic ultrasound-guided pancreatic cyst ablation with ethanol and paclitaxel. Endoscopy 2014;46:457-64.

49 Allen BC, Chen MY, Childs DD, et al. Imaging-guided radiofrequency ablation of cystic renal neoplasms. AJR Am J Roentgenol 2013;200:1365-9.

50 Yoon HM, Baek JH, Lee JH, et al. Combination therapy consisting of ethano and radiofrequency ablation for predominantly cystic thyroid nodules. AJNR Am J Neuroradiol 2013;35:582-6.

51 Gaidhane M, Smith I, Ellen K, et al. Endoscopic ultrasound-guided radiofrequency ablation (EUS-RFA) of the pancreas in a porcine model. Gastroenterol Res Pract 2012;2012:431451.

52 Pai M, Habib N, Senturk H, et al. Endoscopic ultrasound guided radiofrequency ablation, for pancreatic cystic neoplasms and neuroendocrine tumors. World J Gastrointest Surg 2015;7:52-9.

53 Spiliotis JD, Datsis AC, Michalopoulos NV, et al. High operative risk of cool-tip radiofrequency ablation for unresectable pancreatic head cancer. J Surg Oncol 2007;96:89-90.

54 Matsui Y, Nakagawa A, Kamiyama Y, et al. Selective thermocoagulation of unresectable pancreatic cancers by using radiofrequency capacitive heating. Pancreas 2000;20:14-20.

$55 \mathrm{Kim} \mathrm{HJ}$, Seo DW, Hassanuddin A, et al. EUS-guided radiofrequency ablation of the porcine pancreas. Gastrointest Endosc 2012;76:1039-43.

56 Elias D, Baton 0, Sideris L, et al. Necrotizing pancreatitis after radiofrequency destruction of pancreatic tumours. Eur J Surg Oncol 2004;30:85-7.

57 Siriwardena AK. Radiofrequency ablation for locally advanced cancer of the pancreas. J Pancreas 2006;7:1-4.

58 Gage AA, Baust JG. Cryosurgery-a review of recent advances and current issues. Cryo Letters 2002;23:69-78.

59 Xu K, Niu L, Yang D. Cryosurgery for pancreatic cancer. Gland Surg 2013;2:1.

60 Ohno K, Nelson LR, Mitooka K, et al. Transplantation of cryopreserved human corneas in a xenograft model. Cryobiology 2002;44:142-9.

$61 \mathrm{Xu} \mathrm{KC}$, Niu LZ, Hu YZ, et al. Combination of cryosurgery and 125-iodine seed implantation for treatment of locally advanced pancreatic cancer. Technol Cancer Res Treat 2007:6:467-8.

62 Arcidiacono PG, Carrara S, Reni M, et al. Feasibility and safety of EUS-guided cryothermal ablation in locally advanced pancreatic cancer. Gastrointest Endosc 2012;76:1142-51.

63 Richardson AJ, Laurence JM, Lam VW. Transarterial chemoembolization with irinotecan beads in the treatment of colorectal liver metastases: systematic review. J Vasc Interv Radiol 2013;24:1209-17.

64 Yagublu V, Caliskan N, Lewis AL, et al. Treatment of experimental pancreatic cancer by doxorubicin-, mitoxantrone-, and irinotecan-drug eluting beads. Pancreatology 2013;13:79-87.

65 Kotoyan R, Metzger T, Tatum C, et al. Hepatic arterial therapy with drug-eluting beads in the management of metastatic pancreatic carcinoma to the liver: a multi-institutional registry. J Oncol 2012;2012:168303.

66 Karaca C, Cizginer S, Konuk Y, et al. Feasibility of EUS-guided injection of irinotecan-loaded microspheres into the swine pancreas. Gastrointest Endosc 2011;73:603-6.

67 Di Matteo F, Martino M, Rea R, et al. EUS guided Nd:YAG laser ablation of normal pancreatic tissue: a pilot study in pig model. Gastrointest Endosc 2010;72:358-63. http://dx.doi.org/10.1016/j.gie.2010.02.027

68 Kim YH, Saini S, Sahani D, et al. Imaging diagnosis of cystic pancreatic lesions: pseudocyst versus nonpseudocyst. RadioGraphics 2005;25: 671-685. 